\title{
Should known allergy status be included as a medication administration 'right'?
}

\author{
Manfred Mortell
}

\begin{abstract}
This article employs a paediatric case study, involving a 3-year-old child who had an anaphylactic reaction that occurred as a result of the multidisciplinary team's failure to identify and acknowledge the patient's documented 'known allergy' status. It examines and reconsiders the ongoing healthcare dilemma of medication errors and recommends that known allergy status should be considered the second medication administration 'right' before the prescribing, transcribing, dispensing and administration of any drug. Identifying and documenting drug allergy status is particularly important when caring for paediatric patients, because they cannot speak for themselves and must rely on their parents, guardians or health professionals as patient advocates. The literature states that medication errors can be prevented by employing a 'rights of medication administration' format, whether that be the familiar ' 5 rights' or a more detailed list. However, none of these formats specify known allergy status as a distinct 'right'. The medication safety literature is also found wanting in respect of the known allergy status of the patient. When health professionals employ a medication administration rights format prior to prescribing, transcribing, dispensing or administering a medication, the "known allergy status' of the patient should be a transparent inclusion.
\end{abstract}

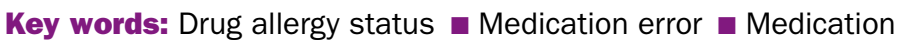
administration $\mathbf{\text { Patient safety }}$ were potentially subjected to at least one ME a day, taking into account near misses, and that not all detected errors were reported (Aspden et al, 2007). Other studies have also indicated that up to $30 \%$ of patients will experience a harmful event such as ME or wrong procedures due to incorrect patient identification during their hospitalisation (Fowler et al, 2008; Griffin and Classen, 2008). A seminal study by Wilson et al (1999) observed that human error was a significant factor, with $81 \%$ of harmful events associated with human factors, such as non-caring attitudes and behaviour, and a lack of knowledge. Vincent et al (2001) categorised $48 \%$ of these harmful occurrences as preventable, which has been supported by other studies (Rex et al, 2000; Regenbogen et al, 2007). In the classic Harvard medical practice study, Leape and colleagues (1991) found that 1 in 4 of these harmful events was the result of negligence and $58 \%$ were deemed preventable.

Some of the most common types of harmful events that resulted in patient mortality involved administration of a drug to patient with a known allergy to that drug or a similar medication. Supplementary reports stated that 1 in 10 patients experience medication-related harm, comparably involving a documented known allergy status (Barker et al, 1962; Bates et al, 1995; Bond et al, 2002; Rothschild et al, 2005). The Joint Commission International (JCI) publishes revised National Patient Safety Goals each year to assist healthcare organisations to improve patient safety; there are specific goals relating to improving medication administration and patient identification (JCI, 2019).

This article considers the ongoing issue of $\mathrm{ME}$ in general and in particular the prescribing and administration of drugs to a patient with a known allergy. It considers a case involving a young child who had an anaphylactic reaction that occurred as a result of the multidisciplinary team's failure to identify and acknowledge the patient's documented 'known allergy' status.

\section{Background \\ Medication errors}

The phenomenon of ME is among the most frequent and widespread of healthcare-related mistakes that cause iatrogenic patient harm (Rothschild, 2005; Aspden et al, 2007; Dhawan et al, 2017). ME have also been clarified as any preventable event that may cause or lead to patient harm while the medication is in the control of the health professional, patient or consumer (Leape, 2005; da Silva and Krishnamurthy, 2016). These potentially harmful events may be related to professional practice, healthcare products, procedures and systems (National Coordinating 